\title{
The assessment and outcomes of human leukocyte antigen mismatches of lung transplantation in Korean patients
}

\author{
Ha Eun Kim¹, Jin Gu Lee ${ }^{1}$, Song Yee Kim², Moo Suk Park ${ }^{2}$, Hyo Chae Paik ${ }^{1}$ \\ ${ }^{1}$ Department of Thoracic and Cardiovascular Surgery, Severance Hospital, Seoul, Korea \\ ${ }^{2}$ Division of Pulmonology, Department of Internal Medicine, Severance Hospital, Seoul, Korea
}

Background: The human leukocyte antigen (HLA) system is an important determinant in solid organ transplantation for allocation. In lung transplantation, HLA compatibility is not included in the lung allocation score system and is not considered when placing donor allografts for lung transplantation. However, multiple studies have suggested that HLA matching affect the outcomes of lung transplantation. This study evaluated the current status of assessment, prevalence, and effects of HLA crossmatches in lung transplantation in Korean patients using nationwide multicenter registry data.

Methods: Two hundred and twenty patients who received lung transplantation at six tertiary hospitals in South Korea between March 2015 and December 2019 are retrospectively reviewed.

Results: Complement-dependent cytotoxic crossmatch (CDC-XM) for T-lymphocyte was performed in 208 patients (94.5\%) and for B-lymphocyte was performed in 154 patients (70.0\%). The flow-cytometric crossmatch for T-lymphocyte was performed in 125 patients $(56.8 \%)$ and for B-lymphocyte was performed in 124 patients $(56.4 \%)$. Among them, nine patients (4.1\%) showed T-cell and/or B-cell mismatches. The incidences of postoperative complications in mismatched patients including primary graft dysfunction, acute rejection, and bronchiolitis obliterans were not significant compared to patients without mismatches. Meanwhile, Kaplan-Meier analyses showed poorer overall survival in patients with mismatch in CDC-XM for T-lymphocyte $(P=0.037)$ and for $B$-lymphocyte $(P=0.085)$ compared to patients without mismatches. The median follow-up was 246.8 days for patients without mismatches and 73.3 days for patients with mismatches.

Conclusions: HLA compatibility is important determinants of lung transplant survival, even with the small number of mismatched patients with relatively short follow-up period. HLA crossmatch should be treated considerably in lung transplantation.

Corresponding author: Jin Gu Lee

E-mail: csjglee@yuhs.ac

(c) The Korean Society for Transplantation

This is an Open Access article distributed under the terms of the Creative Commons Attribution Non-Commercial License (http://creativecommons.org/licenses/by-nc/4.0/) which permits unrestricted non-commercial use, distribution, and reproduction in any medium, provided the original work is properly cited. 